\title{
Change Detection in Bitemporal Remote Sensing I mages by using Feature Fusion and Fuzzy C-Means
}

\author{
Xin Wang ${ }^{1}$, Jing Huang ${ }^{2}$, Yanli Chu ${ }^{1}$, Aiye Shi ${ }^{1, *}$, and Lizhong $\mathrm{Xu}^{1}$ \\ ${ }^{1}$ College of Computer and Information, Hohai University \\ Nanjing, Jiangsu 211100 - China \\ [e-mail: 20100074@hhu.edu.cn] \\ ${ }^{2}$ Business School of Hohai University \\ Nanjing, Jiangsu 211100, China \\ *Corresponding author: Aiye Shi
}

Received June 14, 2017; revised September 17, 2017; accepted November 2, 2017; published April 30, 2018

\begin{abstract}
Change detection of remote sensing images is a profound challenge in the field of remote sensing image analysis. This paper proposes a novel change detection method for bitemporal remote sensing images based on feature fusion and fuzzy c-means (FCM). Different from the state-of-the-art methods that mainly utilize a single image feature for difference image construction, the proposed method investigates the fusion of multiple image features for the task. The subsequent problem is regarded as the difference image classification problem, where a modified fuzzy c-means approach is proposed to analyze the difference image. The proposed method has been validated on real bitemporal remote sensing data sets. Experimental results confirmed the effectiveness of the proposed method.
\end{abstract}

Keywords: Remote sensing image, change detection, feature fusion, fuzzy c-means

This work is supported by the Fundamental Research Funds for the Central Universities (Grant No. 2018B16114), National Natural Science Foundation of China (Grant No. 61603124), Six Talents Peak Project of Jiangsu Province (Grant No. XYDXX-007), and 333 High-Level Talent Training Program of Jiangsu Province. 


\section{Introduction}

In remote sensing, change detection refers to automatically detecting the land-cover changes between a set of remote sensing images acquired over the same geographical area at different observation times [1-3]. It has been applied to a variety of domains, such as urban planning, agriculture surveys, environmental protection, and disaster management [4-6].

In the literature, numerous algorithms have been proposed to identify the changes between bitemporal images [7-13]. These changes detection methods generally consist of two main parts: 1) difference image construction; and 2) classification. Difference image construction, the core of change detection, aims to producing a diffrence image to indicate the extent of the changes. Various of image features have been used for difference image generation according to different requirements and conditions. For instance, Liu et al. [9] used the spectral information for detection multiple changes in hyperspectral images. Lei et al. [10] captured the spatial contextual information for transition detection on remotely sensed images. Wu et al. [11] computed the land cover change by analyzing the texture feature of remotely sensed images. Li and Narayanan [12] selected the shape feature to detect the change information of the lake. Rowe and Grewe [13] utilized the linear feature in remotely sensed images to detect changes. These algorithms have obtained the change detection results. However, they cannot achieve excellent performance due to the utilization of a single type of feature information. Actually, abundances of image features can be integrated to strengthen the performance. However, as far as we know, the computational time increases when increasing the number of image characteristics. Therefore, in this paper we are merely tempted to combine two separate but efficient features for computing difference images, i.e., the gray feature and the texture feature. Through the fused features, the changes will be highlighted, and a robust difference image can be obtained.

The difference images computed above are then used as input for the next part of classification. In recent years, different techniques have been proposed for the difference image classification. For example, Neagoe et al. [14] proposed to use artificial nueral networks for change classification tasks in remote sensing images. Volpi et al. [15] used support vector machines for very high resolution image change detection. Ding et al. [16] presented a sparse hierarchical clustering approach to distinguish between changed and unchanged classes. Besides the above change classification approaches, in the last years, there have been proposed many techniques based on fuzzy c-means (FCM). As is known, c-means method is a commonly used image clustering method. Compared with the traditional hard c-means method, FCM is an improved algorithm [17]. In our real life, there is often no obvious dividing line between different classes. Based on this, FCM introduces the concept of membership degree to describe the uncertainty of objects in different classes, which can make the similarity between objects in the same class largest and the similarity between objects in different classes smallest. Such fuzzy clustering method can reflect the real world more objectively and make up for the defects of the hard c-means method, so it gradually becomes the mainstream of cluster analysis. Due to the good clustering effect of FCM, many researchers have applied it to change detection in remotely sensed images. For instance, Ghost et al. [18] used FCM for unsupervised change detection. Singh et al. [19] combined principal component analysis with hybrid genetic FCM to detect changes of the bitemporal remotely sensed images. Although FCM has been applied to change detection in remote sensing images, direct utilization of it still has some problems. Specifically, the main principle of FCM clustering method is to minimize the objective function by iteration, which usually has slow convergence speed. At the same time, the clustering effect of FCM depends on the selection of the initial values. 
Unsuited initial clustering center may affect the convergence effectiveness and speed of the algorithm. To overcome these two limitations of FCM, we propose an improved fuzzy c-means method to detect the changed and unchanged classes of remote sensing images. First, it modifies the membership degree of the sample points to speed up the convergence speed and minimizes the objective function to improve the accuracy of change detection. At the same time, it selects the initial clustering centers based on the maximum and minimum values in the difference image, which can reduce the probability of local extreme values and further improve the convergence speed.

To this end, a novel change detection in bitemporal remote sensing images based on feature fusion and fuzzy c-means is proposed. The proposed approach is validated on real data sets. The experimental results confirm the effectiveness the proposed method in performing change detection in bitemporal remote sensing images.

The remainder of this paper is organized as follows. Section 2 elaborates the proposed change detection framework. Section 3 reports the experimental results on some real data sets. Finally, conclusions are drawn in Section 4.

\section{Proposed Method}

In this section, an effective framework for change detection in bitemporal remote sensing images is proposed. The unique contributions that can distinguish the proposed work from existing works are twofold. First, a feature fusion based method is presented for difference image construction, which enables it to improve the detection performance based on a single image feature. Second, an improved FCM method is developed for difference image classification, which can not only accelerate the convergence speed, but also improve the effectiveness of change detection. The specific description of these twofold is given as below.

\subsection{Difference Image Construction based on Feature Fusion}

Difference image construction is the first key part in the process of change detection in remote sensing images. In this paper, to overcome the deficiency of insufficient information when constructing the difference image based on a single image feature, we propose to generate the difference image by combining the gray and texture characteristics of remote sensing images.

Let $I_{1}$ and $I_{2}$ be the bitemporal remote sensing images. First, the gray difference image $G$ of $I_{1}$ and $I_{2}$ is calculated by

$$
G(i, j)=\left|I_{1}(i, j)-I_{2}(i, j)\right|, \quad(1 \leq i \leq M, 1 \leq j \leq N)
$$

where $I_{1}(i, j)$ and $I_{2}(i, j)$ represent the gray values of $I_{1}$ and $I_{2}$ at position $(i, j)$, respectively. $M$ and $N$ represent the image's width and height.

Second, we calculate the texture difference image of $I_{1}$ and $I_{2}$ as follows. Haralick et al. [20] defined 14 types of texture feature statistics. Here, we select four kinds, namely, energy, contrast, correlation and entropy, to calculate the texture feature by considering both efficiency and effectiveness of the method. Taking the first temporal image $I_{1}$ as an example, we first calculate the above four texture feature statistics for it. By sliding a window with the size of $7 \times 7$, the step of one pixel, and the direction of $0^{\circ}, 45^{\circ}, 90^{\circ}$ and $135^{\circ}$ on $I_{1}$, we can get four sets of texture feature statistics at different directions. Then, the statistics of these four scanning directions are combined together, and the ultimate texture feature statistics of image 
$I_{1}$, namely, energy matrix $A_{1}$, contrast matrix $C_{1}$, correlation matrix $R_{1}$ and entropy matrix $E_{1}$ are obtained. Similarly, the energy matrix $A_{2}$, contrast matrix $C_{2}$, correlation matrix $R_{2}$ and entropy matrix $E_{2}$ for the second temporal image $I_{2}$ can also be gotten.

Based on the calculated four sets of texture feature statistics, we can subsequently obtain four texture difference matrices of $I_{1}$ and $I_{2}$. Taking the energy difference matrix $A$ as an example, it is defined as

$$
A(i, j)=\left|A_{1}(i, j)-A_{2}(i, j)\right|, \quad(1 \leq i \leq M, 1 \leq j \leq N)
$$

Similarly, the contrast difference matrix $C$, correlation difference matrix $R$ and entropy difference matrix $E$ of $I_{1}$ and $I_{2}$ can be obtained.

The superposition of the above four difference matrices is taken as the ultimate texture difference image $T$. Note that, to make the gray difference image $G$ and the texture feature difference image $T$ have the same value interval, it is necessary to normalize the texture difference image $T$ as

$$
T=\frac{T}{T_{\max }} \times 255
$$

where $T_{\max }$ is the maximum value of $T$.

At last, the ultimate difference image $X$ is defined as

$$
X(i, j)=\frac{1}{2} G(i, j)+\frac{1}{2} T(i, j), \quad(1 \leq i \leq M, 1 \leq j \leq N)
$$

As can be seen, the obtained difference image $X$ contains both gray and texture information of the bitemporal remote sensing images, which will be used as the input of our improved fuzzy c-means method to get the final change detection result.

\subsection{Improved FCM for Difference Image Classification}

Compared with the traditional hard c-means method, FCM introduces the concept of membership degree to realize flexible classification. The membership degree refers to the degree of an object belonging to a set, and can be expressed by a function $u_{\mathrm{A}}(x)$, where $x$ represents the possibility that the object may belong to class $\mathrm{A}$. The value of $u_{\mathrm{A}}(x)$ is in the range $[0,1]$. The higher the value of membership degree is, the greater the likelihood that the object belongs to the set is.

The basic principle of the FCM clustering algorithm is to obtain the optimal solution by minimizing an objective function $J$, and the main realization process is to adjust the clustering center and update the membership degree matrix through iterative operation. The objective function $J$ is defined as 


$$
J=\sum_{k=1}^{n} \sum_{i=1}^{c}\left(u_{i k}^{m}\left\|x_{k}-v_{i}\right\|^{2}\right) \quad \text { Subject to }\left\{\begin{array}{c}
u_{i k} \in[0,1] \\
\sum_{i=1}^{c} u_{i k}=1 \\
0<\sum_{k=1}^{n} u_{i k}<n \\
1 \leq i \leq c ; 1 \leq k \leq n
\end{array}\right.
$$

where $X=\left\{x_{1}, x_{2}, \cdots, x_{n}\right\}$ is the data set to be classified. $x_{k}$ is the kth pixel. $v_{i}$ is the clustering center of the ith class. $n$ is the total number of image pixels. $c(2 \leq c<n)$ is the number of class. $u_{i k}$ represents the membership degree of the $k$ th pixel to the $i$ th class. $\left\|x_{k}-v_{i}\right\|$ represents the distance between the $k$ th pixel and the cluster center of the $i$ th class. $m(1 \leq m \leq \infty)$ is the fuzzy weighting index, which determines the fuzzy degree of the clustering results.

Under the premise of satisfying the constraint condition $\sum_{i=1}^{c} u_{i k}=1$, a new function $F$ is constructed based on $J$ by adding Lagrange multiplier:

$$
F=\sum_{k=1}^{n} \sum_{i=1}^{c}\left(u_{i k}^{m}\left\|x_{k}-v_{i}\right\|^{2}\right)+\sum_{k=1}^{n} \lambda_{k}\left(\sum_{i=1}^{c} u_{i k}-1\right)
$$

where $\lambda_{k}(k=1,2, \cdots, n)$ is the Lagrange multipliers.

The membership degree matrix $u_{i k}$ and the clustering center $v_{i}$ are then calculated by

$$
\begin{gathered}
u_{i k}=\frac{1}{\sum_{j=1}^{c}\left(\frac{\left\|x_{k}-v_{i}\right\|^{2}}{\left\|x_{k}-v_{j}\right\|^{2}}\right)^{\frac{1}{m-1}}}(1 \leq k \leq n ; 1 \leq i \leq c) \\
v_{i}=\frac{\sum_{k=1}^{n} u_{i k}{ }^{m} x_{k}}{\sum_{k=1}^{n} u_{i k}{ }^{m}}(1 \leq k \leq n ; 1 \leq i \leq c)
\end{gathered}
$$

The traditional FCM reflects the real world objectively and makes up for the defects of the hard c-means method, so it gradually becomes the mainstream of cluster analysis. Although FCM has good clustering effect, applying it directly to the change detection in remote sensing images still has some problems. Specifically, the main principle of FCM clustering method is to minimize the objective function by iteration, which usually has slow convergence speed. At the same time, the clustering effect of FCM depends on the selection of the initial values. Unsuited initial clustering center may affect the convergence effectiveness and the speed of the algorithm. Therefore, to get better performance of change detection, here we propose an improved fuzzy c-means method.

Considering the sensitivity of FCM to the initial clustering center, we use the pixels with the highest gray value or the lowest gray value in the difference image as the initial clustering centers of the changed class or the unchanged class, respectively. These two pixels have the highest probability to be the changed or unchanged classes, so it can reduce the possibility of 
generating local extremes in the iterative process and speed up the convergence speed. Moreover, to further overcome the problem of slow convergence speed of FCM, we present a novel strategy. Considering that when the distance between a pixel and a class is obviously smaller than that between this pixel and another class at each iteration process, the probability that the pixel belongs to this class is very high. So we modify the membership degree of the pixel to this class to 1. By doing this, the convergence speed of FCM can be accelerated and at the same time, it is possible to make the sample pixels with the obvious class attributes to play an active role in minimizing the objective function and improve the accuracy of the change detection. The modified FCM is implemented as follows.

First, the difference image $X$ is taken as the input of our improved FCM method. Let $c_{1}$ and $c_{2}$ be the changed class and unchanged class, respectively, $v_{1}$ and $v_{2}$ represent the clustering centers of these two classes. The initial clustering centers are defined as

$$
\begin{aligned}
& v_{1}=X_{\text {max }} \\
& v_{2}=X_{\text {min }}
\end{aligned}
$$

where $X_{\max }$ and $X_{\min }$ are the pixels with the maximum or minimum gray values of the difference image, respectively.

In each iteration, we first calculate the membership degrees of all pixels, and then calculate the European distances between the pixels and two clusters respectively

$$
\begin{aligned}
& D_{1}=\left\{d_{11}, d_{12}, \cdots, d_{1 n}\right\} \\
& D_{2}=\left\{d_{21}, d_{22}, \cdots, d_{2 n}\right\}
\end{aligned}
$$

After that, we use the Eq. (7) calculate the distance ratio $K$, and change the membership degrees of $C_{1}$ pixels with the smallest distance ratios to $u_{1 k}=1, u_{2 k}=0$; change the membership degrees of $C_{2}$ pixels with the biggest distance ratios to $u_{1 k}=0, u_{2 k}=1$. Here, $K$ is calculated by

$$
K=\left\{\frac{d_{11}}{d_{21}}, \frac{d_{12}}{d_{22}}, \cdots, \frac{d_{1 n}}{d_{2 n}}\right\}
$$

Subsequently, we use the Eq. (8) to update the clustering centers $v_{1}$ and $v_{2}$. In each iteration, we increase the values of $C_{1}$ and $C_{2}$, so that more pixels are close to the cluster center, which can speed up the convergence of FCM.

\subsection{Summary of proposed algorithm}

For clarity, we summarize the proposed change detection algorithm in Table 1. As illustrated in Table 1, steps 1-5 correspond to the first part of change detection, i.e., the difference image construction, while steps 6-12 correspond to the second part of change detection, i.e., the difference image classification. Based on these two improved parts, our proposed method can achieve promising change detection performance for bitemporal remote sensing images. 
Table 1. Complete description of proposed change detection algorithm.

Input: Bitemporal remote sensing images $I_{1}$ and $I_{2}$.

Step 1. Preprocess $I_{1}$ and $I_{2}$ by radiometric correction and geometric correction;

Step 2. Calculate the gray difference image $G$ by Eq. (1);

Step 3. Calculate four texture difference matrices by Eq. (2);

Step 4. Superpose four difference matrices to get the ultimate texture difference image $T$ and normalize $T$ by Eq. (3);

Step 5. Calculate the ultimate difference image $X$ by Eq. (4);

Step 6. Initialize the cluster center $v_{1}^{0}$ and $v_{2}^{0}$ of the difference image $X$ by Eq. (9) and Eq. (10);

Step 7. Calculate the membership degree matrix $u_{i k}$ of all pixels in $X$ by Eq. (7);

Step 8. Calculate the European distances between all pixels in $X$ and two clusters, respectively.

Step 9. Calculate the distance ratio $K$ by Eq. (13) and change the membership degrees of $C_{1}$ pixels with the smallest distance ratios to $u_{1 k}=1, u_{2 k}=0$; change the membership degrees of $C_{2}$ pixels with the biggest distance ratios to $u_{1 k}=0, u_{2 k}=1$;

Step 10. Update the clustering centers $v_{1}^{t}$ and $v_{2}^{t}$ by Eq. (8);

Step 11. If $t>T$ or $\frac{\left\|v_{1}^{t}-v_{1}^{t-1}\right\|}{v_{1}^{t-1}}<\varepsilon$ and $\frac{\left\|v_{2}{ }^{t}-v_{2}{ }^{t-1}\right\|}{v_{2}^{t-1}}<\varepsilon$, stop the iteration and output the membership degree matrix; otherwise, update $v_{i}=v_{i}^{t}(1 \leq i \leq 2), t=t+1$, and increase the values of $C_{1}$ and $C_{2}$, then go to Step 7;

Step 12. Utilize the final membership degree matrix $u_{i k}$ to determine the class of all the pixels: if $u_{1 k}>u_{2 k}(k=1,2, \cdots, n)$, the pixel $k$ belongs to the changed class; otherwise, it belongs to the unchanged class.

Output:Final change detection results.

\section{Experimental Results and Analysis}

\subsection{Experimental Setup}

To verify the performance of the proposed change detection algorithm, a set of experiments have been conducted. All experiments are implemented on a PC with an Intel Core $1.70 \mathrm{GHz}$ processor and 4.00 GB RAM. The programming platform is MATLAB R2013b. In this section, we present the experimental results on two real datasets.

The first is the Mexico dataset. It consists of two images with the size of $512 \times 512$ of Mexico suburb, which are acquired by the Landsat 7 ETM+ (Enhanced Thematic Mapper Plus) sensor in April 2000 ("before”) (as shown in Fig. 1 (a)) and May 2002 ("after") (as shown in Fig. 1 (b)). In that period, the main change is caused by the fire. The ground reference shown in Fig. 1 (c) was obtained by manual annotation, which combines expert knowledge and prior information. 


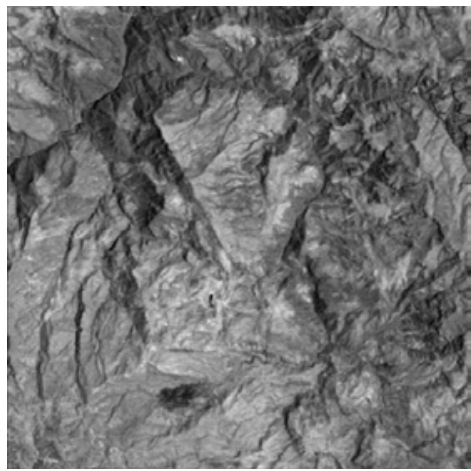

(a)

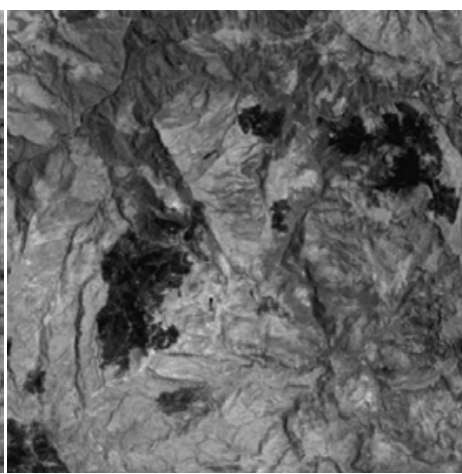

(b)

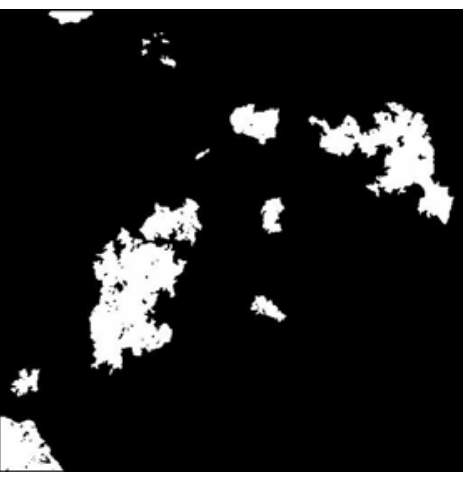

(c)

Fig. 1. Mexico dataset and its ground truth data.

(a) Image captured in April 2000. (b) Image captured in May 2002. (c) Groud truth data.

The second is the Sardinia dataset. It consists of two images with the size of $412 \times 300$ of Sardinia, which are acquired by the Landsat-5 TM (Thematic Mapper) sensor in September 1995 ("before”) (as shown in Fig. 2 (a)) and July 1996 ("after”) (as shown in Fig. 2 (b)). In that period, the main change is caused by the rise of the lake water level. The ground reference shown in Fig. 2 (c) was also produced via manual marking by the combination of specialistic knowledge and surface prior information.

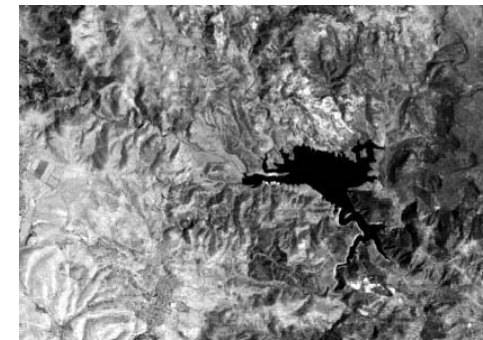

(a)

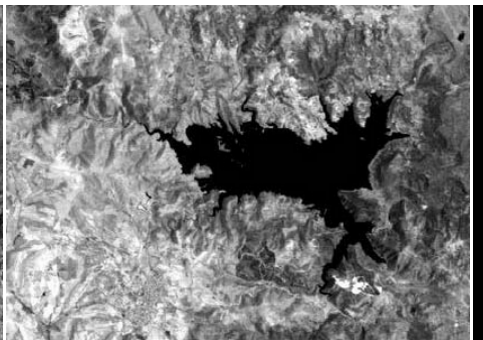

(b)

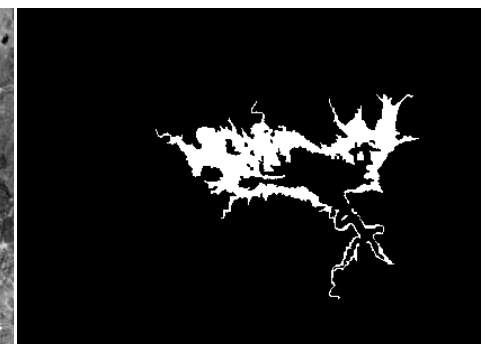

(c)

Fig. 2. Sardinia dataset and its ground truth data.

(a) Image captured in September 1995. (b) Image captured in July 1996. (c) Groud truth data.

We compare our proposed change detection method with several classical algorithms, including the expectation maximization (EM) method [21], the hard c-means (HCM) method [22], and the traditional FCM method [23].

Besides, in order to carry out the quantitative evaluation, we compute five criterions [24-27] by comparing the resulting change detection map with the ground truth: 1) False alarms (FA); 2) Missed alarms (MA); 3) Overall errors (OE) ; 4) Overall accuracy (OA); 5) Kappa statistics (Kappa). Thereinto, FA denotes the number of unchanged pixels detected as changed pixels. MA denotes the number of changed pixels detected as unchanged pixels. Generally, the fewer FA value means better noise immunity, and the fewer MA value means greater identification of changed areas.

Based on FA and MA, we can calculate OE, which denotes the total number of wrongly detected pixels. It is computed by using:

$$
\mathrm{OE}=\mathrm{FA}+\mathrm{MA}
$$


Obviously, the fewer OE value means better change detection performance.

Furthermore, the overall accuracy, OA, can be calculated by:

$$
\mathrm{OA}=\frac{\mathrm{TP}+\mathrm{TN}}{\mathrm{TP}+\mathrm{TN}+\mathrm{FA}+\mathrm{MA}}
$$

where TP denotes the number of changed pixels detected as changed pixels. TN denotes the number of unchanged pixels detected as unchanged pixels. Contrary to OE, the higher OA value means better change detection result.

At last, Kappa can be computed through:

$$
\text { Kappa }=\frac{\text { OA-PRE }}{1-P R E}
$$

where

$$
\mathrm{PRE}=\frac{(\mathrm{TP}+\mathrm{FA}) \cdot(\mathrm{TP}+\mathrm{MA})}{(\mathrm{TP}+\mathrm{TN}+\mathrm{FA}+\mathrm{MA})^{2}}+\frac{(\mathrm{TN}+\mathrm{FA}) \cdot(\mathrm{TN}+\mathrm{MA})}{(\mathrm{TP}+\mathrm{TN}+\mathrm{FA}+\mathrm{MA})^{2}}
$$

For Kappa, the higher it is, the better the change detection performance is.

\subsection{Results on Mexico Dataset}

By applying the EM, HCM, FCM and our proposed method to the Mexico dataset respectively, we can get their change detection results. In the following, the performance of the four methods are shown and analyzed.

First, we evaluate the change detection results quanlitatively. The quanlitative results are shown as change maps (binary maps). Black pixels on a change map represent the unchanged areas, and white pixels denote the changed areas. The change maps pf the four methods are shown in Fig. 3. Fig. 3 (a) shows the change detection result obtained by the EM method. Compared with the ground truth image (see Fig.1 (c)), the EM method produces a few false alarms. However, it has many missed alarms. Fig. 3 (b) shows the change detection result obtained by the HCM method. It is obvious that the HCM not only produces too many false alarms, but also has a large number of missed alarms. Fig. 3 (c) and (d) compares the performance of the FCM and our method. Due to the introduction of the concept of membership degree, the FCM method (see Fig.3 (c)) and our proposed method (see Fig.3 (d)) exhibit better visualization results, compared with the EM and HCM algorithms. It is can be seen that both of the FCM and our method keep changed areas detected well. Furthermore, our method produces smoother unchanged regions compared with the FCM. It means that our method produces fewer false alarms and it suppresses the bad effects of noise to greater extent than the FCM. This advantage of our method over FCM comes from the multifeature fusion in which both gray and texture information is utilized for improving its noise immunity. 


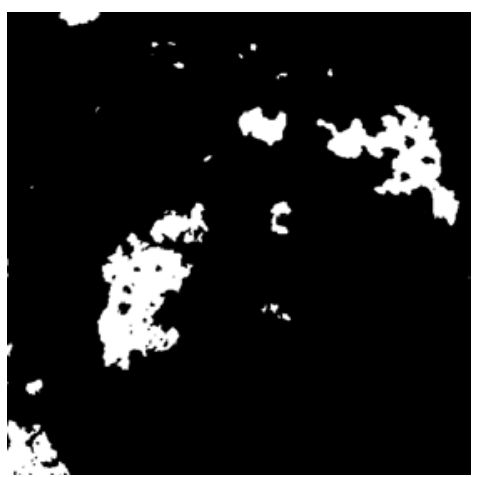

(a)

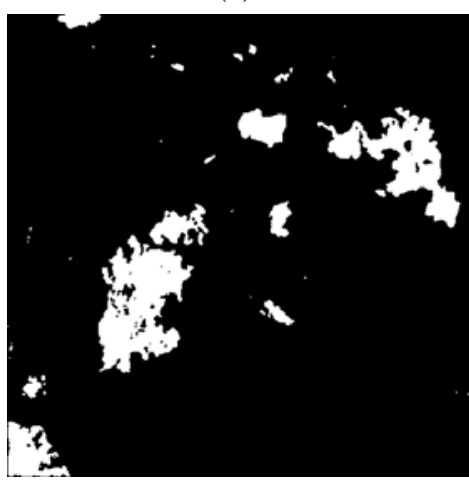

(c)

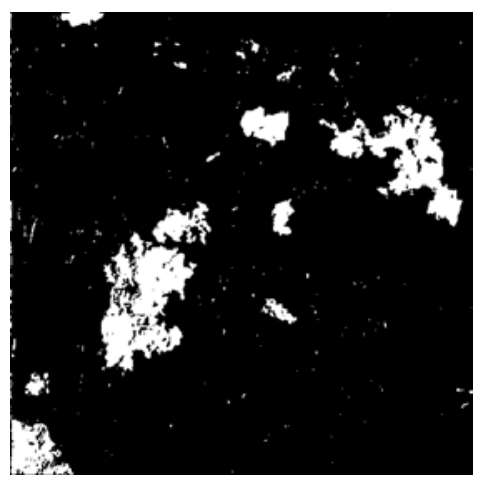

(b)

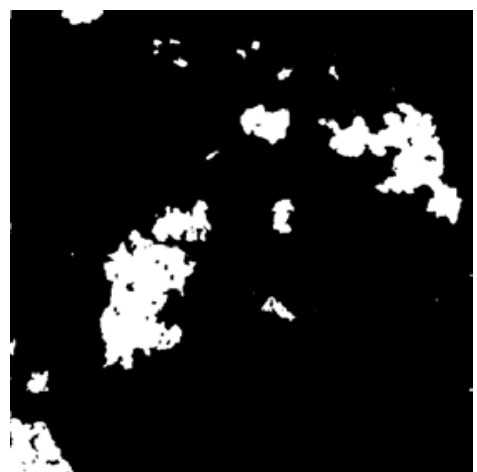

(d)

Fig. 3. Change maps of Mexico dataset. (a) Change map resulting from the EM. (b) Change map resulting from the HCM. (c) Change map resulting from the FCM. (d) Change map resulting from our method.

Second, the quantitative evaluations of the change detection results are given. The flase alarms, missed alarms, overall errors, and Kappa values are given in Table 2. The best results are emphasized as bold. Obviously, our method achieves quite good results. First, it can be seen from Table 2 that the proposed method produces the lowest false alarm (1352), lowest missed alarm (2430), lowest overall error (3782), highest overall accuracy (0.9856), and highest Kappa value (0.9336) compared with the other three methods, which means that our method has the strongest noise immunity and greatest identification ability. Moreover, larger Kappa values of the FCM over EM and HCM are illustrated, which prove further the validity of membership degree. In a word, the quantitative results prove further the great strong noise immunity and good identification of changed areas of our feature fusion and fuzzy c-means based method for change detection in bitemporal remote sensing images.

Table 2. Quantitative comparison of change detection for Mexico dataset ( $512 \times 512$ pixels).

\begin{tabular}{|c|c|c|c|c|}
\hline Algorithms & EM & HCM & FCM & Our method \\
\hline \hline FA & 1418 & 2581 & 1524 & $\mathbf{1 3 5 2}$ \\
\hline MA & 3353 & 3562 & 2523 & $\mathbf{2 4 3 0}$ \\
\hline OE & 4771 & 6143 & 4047 & $\mathbf{3 7 8 2}$ \\
\hline OA & 0.9818 & 0.9766 & 0.9846 & $\mathbf{0 . 9 8 5 6}$ \\
\hline Kappa & 0.8903 & 0.8619 & 0.9122 & $\mathbf{0 . 9 3 3 6}$ \\
\hline
\end{tabular}

Bold Indicates the Best Performance. 
At last, to verify the higher convergence speed of our method compared with the traditional FCM method, we calculate the convergence curves of objective functions of these two algorithms. The results are given in Fig. 4. Fig. 4 (a) is the convergence curve of objective function by FCM. Fig. 4 (b) is the convergence curve of objective function by our method. It is obtained intuitively from Fig. 4 that the FCM converges at the 11th iteration, while our proposed method converges at the 6th iteration. Hence, the convergence speed of our proposed method is faster than the FCM. At the same time, the initial objective function of our method is obviously smaller than that of the FCM. This advantage of our method over FCM comes from our modification of the traditional FCM approach. In conclusion, our proposed method achieve better efficiency compared with the FCM.

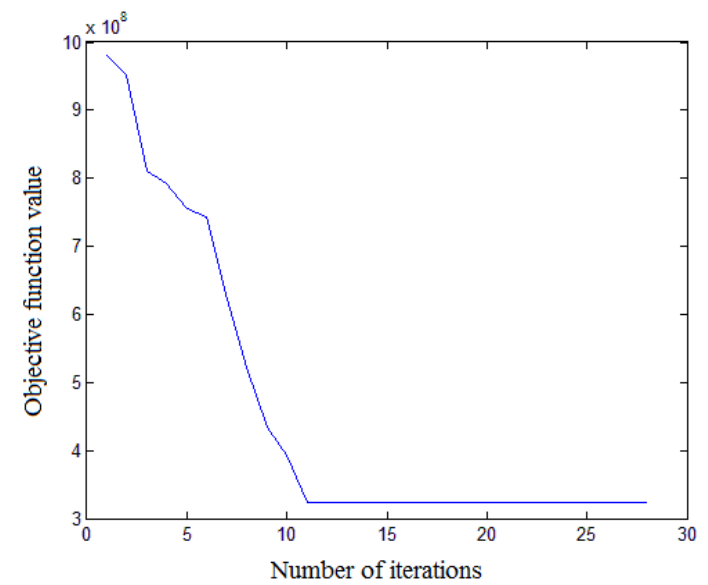

(a)

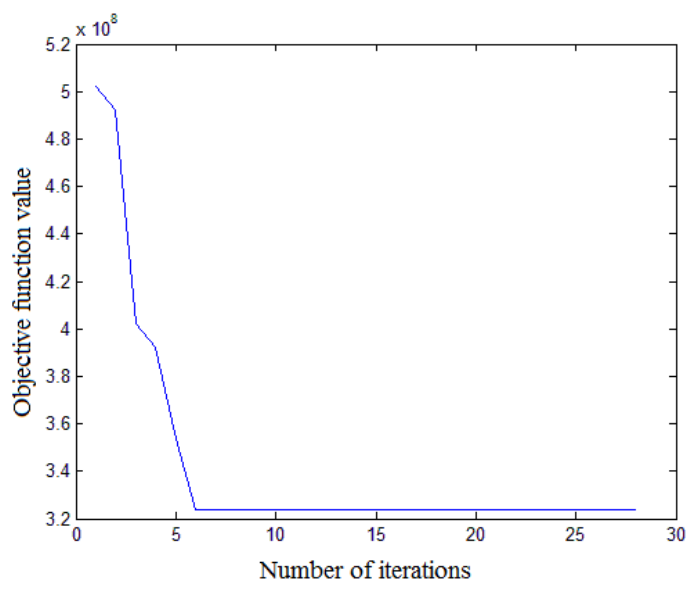

(b)

Fig. 4. Convergence curves of objective function for Mexico dataset. (a) Convergence curve of objective function by FCM. (b) Convergence curve of objective function by our method.

\subsection{Results on Sardinia Dataset}

In this section, the EM, HCM, FCM and our proposed method are respectively applied to the Sardinia dataset, leading to similar results.

First, let us analyze, in detail, the qualitative results of the competing algorithms. Fig. 5 shows the change maps of the four methods. Fig. 5 (a) shows that EM produces fewer false alarms. But it misses a lot of small changed areas. Fig. 5 (b) shows that HCM is not good at resisting noise and preserving edge locations. The results in Fig. 5 (c) show that the FCM keeps changed areas detected a bit better than the EM and HCM. However, compared with the results in Fig. $\mathbf{5}$ (d), the performance of FCM is still inferior. The results in Fig. $\mathbf{5}$ (d) show that our method identifies the changed areas more precisely, particularly small changes. This strong identification derives from the feature fusion and improved fuzzy c-means. In summary, our method has two merits, i.e., good identification of changed areas and strong noise immunity, simultaneously. 


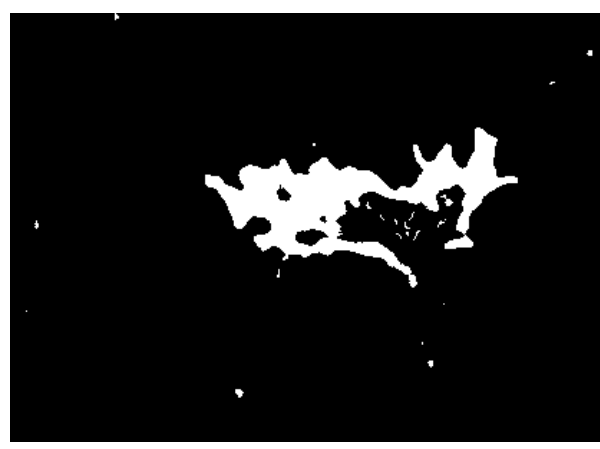

(a)

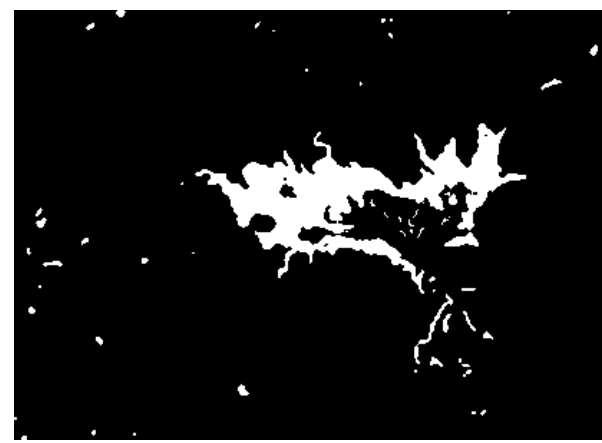

(c)

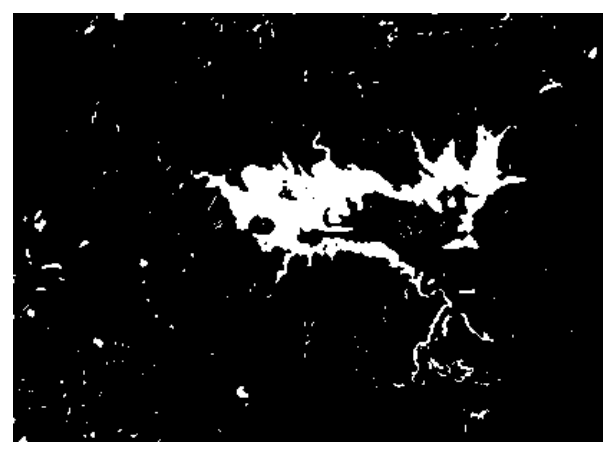

(b)

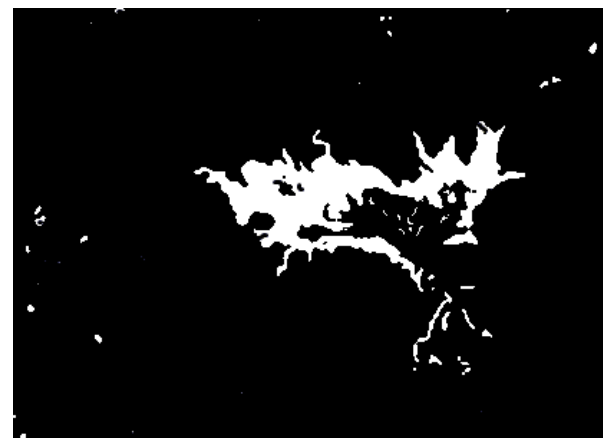

(d)

Fig. 5. Change maps of Sardinia dataset. (a) Change map resulting from the EM. (b) Change map resulting from the HCM. (c) Change map resulting from the FCM. (d) Change map resulting from our method.

Second, the quantitative comparison of change detection for Sardinia dataset is shown in Table 3. The best results are emphasized as bold. From the table, we can see that our method is superior to other methods in terms of missed alarm (759), overall error (1962), overall accuracy (0.9841), and Kappa value (0.8632). In contrast, EM, HCM, and FCM have relatively higher false alarms, higher missed alarms, higher overall errors, lower overall accuracy values, or lower Kappa values.

Table 3. Quantitative comparison of change detection for Sardinia dataset $(412 \times 300$ pixels $)$.

\begin{tabular}{|c|c|c|c|c|}
\hline Algorithms & EM & HCM & FCM & Our method \\
\hline \hline FA & $\mathbf{1 1 9 6}$ & 2637 & 1225 & 1203 \\
\hline MA & 1231 & 1326 & 783 & $\mathbf{7 5 9}$ \\
\hline OE & 2427 & 3963 & 2008 & $\mathbf{1 9 6 2}$ \\
\hline OA & 0.9804 & 0.9679 & 0.9837 & $\mathbf{0 . 9 8 4 1}$ \\
\hline Kappa & 0.8046 & 0.7265 & 0.8427 & $\mathbf{0 . 8 6 3 2}$ \\
\hline
\end{tabular}

Bold Indicates the Best Performance.

Similarly, we ultimately compare the convergence speeds between our method and the traditional FCM method to verify the effectiveness of our modified FCM scheme. The results are given in Fig. 6. As shown in Fig. 6 (a), the FCM converges at the 11th iteration, while while our proposed method converges at the 7th iteration. Therefore, for Sardinia dataset, our proposed method also has a faster convergence speed and a smaller initial value of objective function. Thus, our proposed method indeed has the best performance to detect changes among the four methods. 


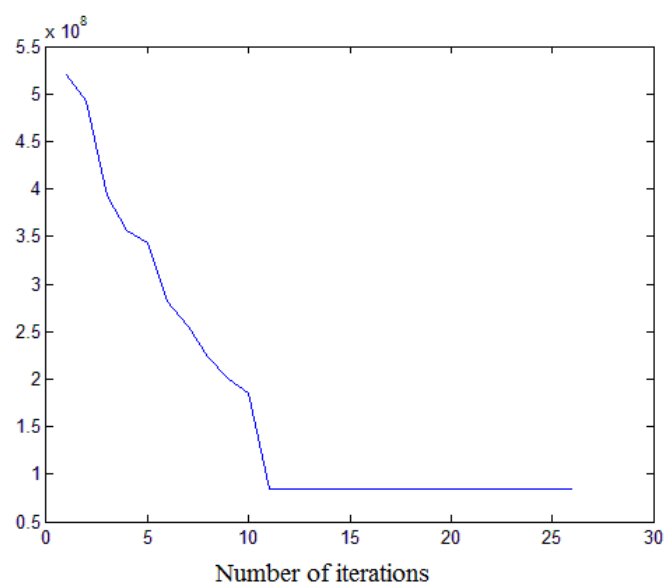

(a)

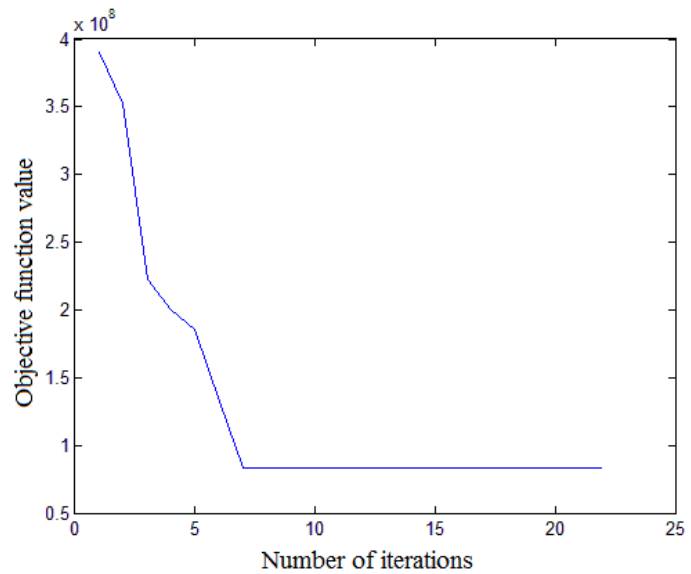

(b)

Fig. 6. Convergence curves of objective function for Sardinia dataset. (a) Convergence curve of objective function by FCM. (b) Convergence curve of objective function by Our method.

\section{Conclusion}

In this paper, a novel method has been proposed to address the hot change detection problem in bitemporal remote sensing images. The main novelties and contributions of the proposed method are as follows: 1 ) it designs an improved approach to construct the difference image for bitemporal remote sensing images, by which the changes are highlighted and a robust difference image is obtained; 2 ) it proposes a modified fuzzy c-means technique to solve the difference image classification problem, through which the convergence speed is accelerated and the accuracy of change detection is improved. Experimental results obtained on real remote sensing images confirmed the effectiveness of the proposed method. Our future work will incorporate some more powerful features, which may further increase the detection performance.

\section{References}

[1] Natalia Sofina and Manfred Ehlers, "Building change detection using high resolution remotely sensed data and GIS," IEEE Journal of Selected Topics in Applied Earth Observations and Remote Sensing, vol. 9, no. 8, pp. 3430-3438, August, 2016. Article (CrossRef Link).

[2] Juan P. Ardila, Wietske Bijker, Valentyn A. Tolpekin and Alfred Stein, "Quantification of crown changes and change uncertainty of trees in an urban environment," ISPRS journal of photogrammetry and remote sensing, vol. 2012, no. 74, pp. 41-55, November, 2012.

Article (CrossRef Link).

[3] Xin Wang, Siqiu Shen, Chen Ning, Fengchen Huang and Hongmin Gao, "Multi-class remote sensing object recognition based on discriminative sparse representation," Applied Optics, vol. 55, no. 6, pp. 1381-1394, June, 2016. Article (CrossRef Link).

[4] Mengxi Xu, Quansen Sun, Yingshu Lu, Fengchen Huang and Chenrong Huang, "A Novel Fast Remote Sensing Targets Detection Model based on KSC-SBOW," International Journal of Signal Processing, Image Processing and Pattern Recognition, vol. 9, no. 3, pp. 341-354, March 2016. Article (CrossRef Link).

[5] Xin. Wang, Guofang Lv and Lizhong Xu, "Infrared dim target detection based on visual attention," Infrared Physics \& Technology, vol. 55, no. 6, pp. 513-521, June, 2012. Article (CrossRef Link). 
[6] Mengxi Xu, Quansen Sun, Zhenyu He and Jianqiang Shi, "Band selection for hyperspectral images based on particle swarm optimization and differential evolution algorithms with hybrid encoding," Journal of Computational Methods in Sciences and Engineering, vol. 16, no. 3, pp. 629-640, October, 2016. Article (CrossRef Link).

[7] Adel Shalaby, Ryutaro Tateishi, "Remote sensing and GIS for mapping and monitoring land cover and land-use changes in the Northwestern coastal zone of Egypt,” Applied Geography, vol. 27, no. 1, pp. 28-41, January, 2007. Article (CrossRef Link).

[8] Shaun Quegan, Thuy Le Toan, Jiong Jiong Yu, Florence Ribbes and Nicolas Floury, "Multitemporal ERS SAR analysis applied to forest mapping," IEEE Transactions on Geoscience and Remote Sensing, vol. 38, no. 2, pp. 741-753, March 2000. Article (CrossRef Link).

[9] Sicong Liu, Lorenzo Bruzzone, Francesca Bovolo and Peijun Du, "Unsupervised Multitemporal Spectral Unmixing for Detecting Multiple Changes in Hyperspectral Images,” IEEE Transactions on Geoscience and Remote Sensing, vol. 54, no. 5, pp. 2733-2748, May 2016. Article (CrossRef Link).

[10] Zhen Lei, Tao Fang, Hong Huo and Deren Li, "Bi-Temporal Texton Forest for Land Cover Transition Detection on Remotely Sensed Imagery,” IEEE Transactions on Geoscience and Remote Sensing, vol. 52, no. 5, pp. 1227-1237, February 2014. Article (CrossRef Link).

[11] Xiaofeng Wu, Fen Yang and Roly Lishman, "Land cover change detection using texture analysis," Journal of Computer Science, vol.6, no.1, pp. 92-100, January, 2010. Article (CrossRef Link).

[12] Jiang Li and Ram M. Narayanan, "A shape-based approach to change detection of lakes using time series remote sensing images,” IEEE Transactions on Geoscience and Remote Sensing, vol. 41, no. 11, pp. 2466-2477, November, 2003. Article (CrossRef Link).

[13] Neil C. Rowe and Lynne L. Grewe, "Change detection for linear features in aerial photographs using edge-finding,” IEEE Transactions on Geoscience and Remote Sensing, vol. 39, no. 7, pp. 1608-1612, July, 2001. Article (CrossRef Link).

[14] Victor-Emil Neagoe, Radu-Mihai Stoica, Alexandru-Ioan Ciurea,Lorenzo Bruzzone and Francesca Bovolo, "Concurrent Self-Organizing Maps for Supervised/Unsupervised Change Detection in Remote Sensing Images” IEEE Journal of Selected Topics in Applied Earth Observations and Remote Sensing, vol. 7, no. 8, pp. 3525-3533, August, 2014. Article (CrossRef Link).

[15] Michele Volpi, Devis Tuia, Francesca Bovolo, Mikhail Kanevski and Lorenzo Bruzzonec, "Supervised change detection in VHR images using contextual information and support vector machines,” International Journal of Applied Earth Observation and Geoinformation, vol. 20, pp. 77-85, Febuary, 2013. Article (CrossRef Link).

[16] Kun Ding, Chunlei Huo, Yuan Xu, Zisha Zhong and Chunhong Pan, "Sparse Hierarchical Clustering for VHR Image Change Detection,” IEEE Geoscience and Remote Sensing Letters, vol. 12, no. 3, pp. 577-581, March, 2015. Article (CrossRef Link).

[17] Nikhil R. Pal and James C. Bezdek, "On cluster validity for the fuzzy c-means model," IEEE Transactions on Fuzzy systems, vol. 3, no. 3, August, 1995. Article (CrossRef Link).

[18] Susmita Ghosh, Niladri Shekhar Mishra and Ashish Ghosh, "Unsupervised change detection of remotely sensed images using fuzzy clustering," in Proc. of IEEE International Conference on Advances in Pattern Recognition, pp. 385-388, February 4-6, 2009. Article (CrossRef Link).

[19] Krishna Kant Singh, AkanshaMehrotra, M.J.Nigam and Kirat Pal, "Unsupervised change detection from remote sensing images using hybrid genetic FCM,” in Proc. of IEEE International Conference on Engineering and Systems, pp. 1-5, April, 2013. Article (CrossRef Link).

[20] Robert Martin Haralick, K. Shanmugam and Its'Hak Dinstein, "Textural features for image classification,” IEEE Transactions on systems, man, and cybernetics, vol. 3, no. 6, pp. 610-621, December, 1973. Article (CrossRef Link).

[21] Ming Hao, Wenzhong Shi, Hua Zhang and Chang Li, "Unsupervised Change Detection With Expectation-Maximization-Based Level Set," IEEE Geoscience and Remote Sensing Letters, vol. 11, no. 1, January, 2014. Article (CrossRef Link).

[22] T. A. Pawar, "Change Detection Approach for Images Using Image Fusion and C-means Clustering Algorithm,” International Journal of Advanced Research in Computer Science and 
Management Studies, vol. 2, no. 10, pp. 303-307, October, 2014. Article (CrossRef Link).

[23] Mustafa Hayri Kesikoğlu, Ü. H Ataseve and C. Özkan, "Unsupervised change detection in satellite images using fuzzy c-means clustering and principal component analysis,” ISPRS-International Archives of the Photogrammetry, Remote Sensing and Spatial Information Sciences, vol. XL-7/W2, no. 7, pp. 129-132, November 2013. Article (CrossRef Link).

[24] Lorenzo Bruzzone and Diego Fernàndez Prieto, “Automatic analysis of the difference image for unsupervised change detection,” IEEE Transactions on Geoscience and Remote sensing, vol. 38, no. 3, pp. 1171-1182, May, 2000. Article (CrossRef Link).

[25] Lu Jia, Ming Li, Peng Zhang and YanWu, "SAR Image Change Detection Based on Correlation Kernel and Multistage Extreme Learning Machine,” IEEE Transactions on Geoscience and Remote sensing, vol. 54, no. 10, pp. 5993-6006, October, 2016. Article (CrossRef Link).

[26] Maoguo Gong, Puzhao Zhang, Linzhi Su and Jia Liu, "Coupled Dictionary Learning for Change Detection From Multisource Data,” IEEE Transactions on Geoscience and Remote sensing, vol. 54, no. 12, pp. 7077-7091, December, 2016. Article (CrossRef Link).

[27] Maoguo Gong, Tao Zhan, Puzhao Zhang and Qiguang Miao, "Superpixel-Based Difference Representation Learning for Change Detection in Multispectral Remote Sensing Images,” IEEE Transactions on Geoscience and Remote sensing, vol. 55, no. 5, pp. 2658-2673, May, 2017. Article (CrossRef Link). 


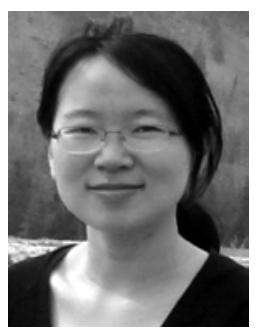

Xin Wang received the Ph.D. degree in Computer Application Technology from Nanjing University of Science and Technology, Nanjing, China, in 2010. She is currently an Associate Professor with the College of Computer and Information, Hohai University, Nanjing, China. She has published more than 50 papers in journals and referred conferences. Her current research interests include image processing, computer vision, and pattern recognition.

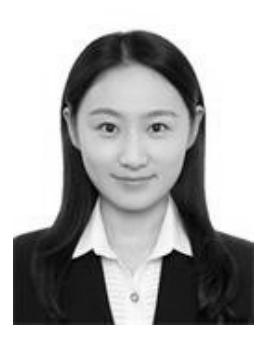

Jing Huang received the Ph.D. degree in agement Science and Engineering from University of Bristol, England, in 2013. She is currently a lecture with the Business School of Hohai University, Nanjing, China. Her current research interests include signal and information processing.

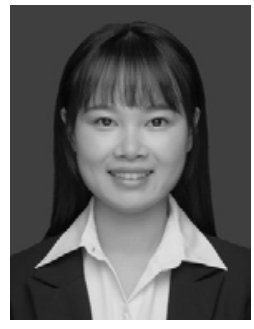

Yanli Chu received the B.S. degree in Communication Engineering from Hohai University, Nanjing, China, in 2014. Now she is working toward the M.S. degree in the College of Computer and Information, Hohai University. Her current research interests include remote sensing image processing.

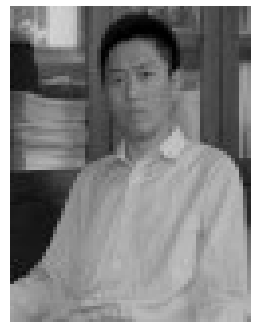

Aiye Shi received the Ph.D. degree in Computer Science from Hohai University, Nanjing, China. He is currently an Associate Professor in the university. He has published more than 30 papers in journals and referred conferences. His current research interests include remote sensing image processing and analysis.

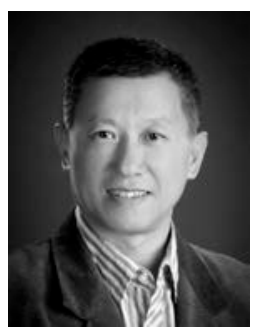

Lizhong Xu received the Ph.D. degree in Information and Communication Engineering from China University of Mining and Technology, Nanjing, China. He is currently a Professor in Hohai university. He has published more than 80 papers in journals and referred conferences. His current research interests include image aciqusition, processing, and analysis. 